\title{
Anti-Aging Medicine for Hair
}

\author{
John cole* and Megan A Cole \\ Cole Hair Transplant Group, Georgia, USA \\ Submission: May 05, 2018; Published: May 24, 2018 \\ *Corresponding author: Cole Hair Transplant Group, Alpharetta, GA 30004, USA, Email: forhair3@me.com
}

\section{Introduction}

Aging is a natural and unavoidable consequence of life; however, the appearance of being "aged" need not follow suit. Indeed, the biology associated with cosmetic aging, the chemical pathways that trigger collagen degradation and isolated areas of hyper pigmentation in the dermis, as well as hair loss and/ or graying in the scalp, is a growing area of research, and as scientists unveil key regulators of the aging cascade, clinicians become better equipped to rejuvenate wrinkled skin and increase scalp hair density. In the context of anti-aging medicine, the hair follicle is uniquely qualified to provide a wealth of knowledge; it is easily accessible, contains multiple distinct adult stem cell populations, and regenerates itself cyclically. Moreover, the hair follicle constitutes a miniorgan with environmental niches established for both quasi-permanent, slow-cycling stem cells and transit-amplifying, highly-proliferative progenitor cells [1]. Areas including the bulge, isthmus, and infundibulum are present in all stages of the hair growth cycle. Unsurprisingly, multiple adult stem cell populations have been identified in these regions, particularly in the outer root sheath region lying directly below the sebaceous gland, or the bulge region. On the other hand, the hair follicle matrix and pre-cortex are only observed during the active growth phase of the hair cycle (anagen) where they serve as reservoirs of rapidly dividing keratinocyte progenitor cells and as differentiation and melanin synthesis centers, respectively.

\section{The Hair Cycle}

The hair cycle begins with a short stage of apoptotic-driven hair follicle regression (catagen) that lasts approximately 2 weeks. During catagen, the deeper, highly proliferative structures of the hair follicle, namely the matrix and the precortex, are lost, while the hair shaft, along with the inner and outer root sheaths, regress up towards the scalp surface. Following this period of regression, the hair follicle enters a state of relative quiescence (telogen); the dermal papilla condenses, and the hair shaft is actively held in place by a specialized junction complex located at the base of the bulge region. The depigmented, fully keratinized telogen hair shaft is referred to as the "club" hair in homage the characteristic morphology of its club-like base. Notably, the secondary hair germ, which contains bulge stem cell-derived progenitor cells that will eventually give rise to the anagen hair bulb, pigmented hairs haft, and inner roots heath, manifests early in telogen and expresses an impressive quantity of circadian clock target genes. Deletion of two such genes, Bmal1 and Clock, delays the onset of anagen in mice without altering the morphological appearance of the hair follicle once growth finally ensues [2]. Interestingly, knock down of the circadian prote in BMAL1 (orPeriod1) significantly prolongs the anagen phase in human hair follicles already in anagen, rendering these two chronobiological proteins potential drug targets for future hair follicle anti-aging medication(s) [3].

Late in telogen, the secondary hair germ is activated, and, following a period of rapid proliferation, elongates distally into the subcutaneous tissue, enveloping the dermal papilla and establishing itself as a matrix of proliferative transit-amplifying cells at the base of the follicle. Cell differentiation programs are reactivated, giving rise to the inner root sheath and hair shaft, and differentiation of melanocyte precursors leading to melanogenesis occurs. Meanwhile, the club hair is shed (exogen) in an independently regulated process. Although the duration of catagen and angered main fairly consistent from one cycle to the next, each telogen becomes progressively longer than the one before; consequently, a progressive asynchrony in hair follicle cycling is observed with age. Additionally, many hair loss disorders (androgenic alopecia, alopecia are ate, telogen effluvium) are characterized by concomitant increases in telogen and reduction sinanagen making the telogen to anagen regulatory path way(s) of particular importance in the rational design of anti-aging therapies. To date, two separate signaling pathways have been linked to hair follicle regeneration and underpin the readiness of the telogen follicle to enter anagen. Competing gradients of their inhibitory signals (bone morphogenetic protein (BMP) and fibroblast growth factor 18(Fgf18)) and stimulatory signals (wingless (Wnt) and Fgf7/10) cycles lightly out of phase with one another, thereby establishing an early refractory telogen follicle and later competent telogen follicle [4]. 


\section{The Wnt/b-catenin Pathway}

The first of the two telogen to anagen regulatory pathways is the canonical Wnt/b-catenin cycle, which is critical for maintaining the bulge stem cells and secondary hair germ cells in their respective undifferentiated states [5]. In the absence of Wnt, the downstream effector molecule, b- catenin, is inactive, and its nuclear targets, Tcf/Lef, interact with corepressor molecules such as Groucho to actively repress gene transcription [6]. Wnt proteins are legends for the Frizzled (Fz) family of cell surface receptors; they undergo substantial posttranslational modification (glycosylation, palmitolation) in the endoplasmic reticulum and are secreted into the extracellular milieuas glycoproteins. Binding of Wnt to their receptor complex composed of $\mathrm{Fz}$ and low-density- lipoprotein-related protein 5/6 (Lrp5/6) induces b-catenin-directed transcriptional regulation of target genes. b-catenin is a dual function protein, acting as both an adherens junction-associated protein and a transcriptional co-activator. When the $\mathrm{Fz}$ receptor site is vacant, cytoplasmic b- catenin undergoes targeted proteosomal degradation via the b-catenin destruction complex, an assembly of axin, adenomatosis polyposis coli (APC), protein phosphatase 2A (PP2A), glycogen synthase kinase 3 (GSK3), and casein kinase 1a (CK1a). GSK3 and CK1 a sequentially phosphor late a set of conserved residues in the $\mathrm{N}$-terminus of $\mathrm{b}$-catenin, marking it for ubiquitination and subsequent degradation. However, association of Wnt with the Fz/Lrp5/6 receptor disrupts the b-catenin destruction complex, stabilizing b-catenin within the cytoplasm where it accumulates, travels into the nucleus, and associates with DNA-binding proteins of the Tcf/Lef family [7] (Figure 1).

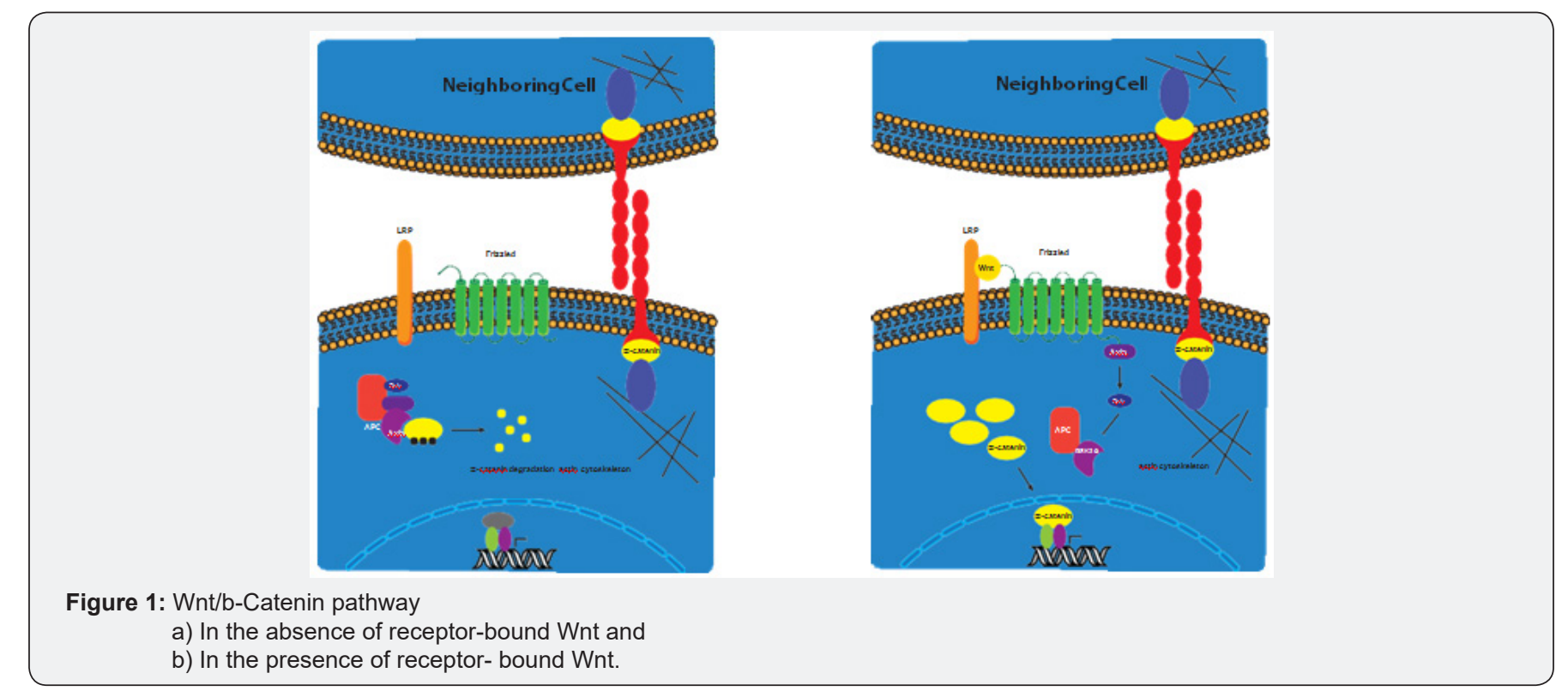

\section{The BMP Pathway}

The second regulatory pathway known to drive hair follicles from telogen to anagen involves crosstalk between the mesenchymal and endothelial compartments and is governed by BMP. In telogen, fibroblasts in the dermal papilla and keratinocytes in the secondary hair germ express BMP4. Additionally, fibroblasts in the dermis express BMP2. Binding of BMP to bone morphogenetic receptor type 1A (BMPR-1A), which is selectively expressed in the secondary hair germ during early (refractory) telogen, inhibits Wnt expression and its downstream effectors [8]. Noggin expression in the hair follicle epithelium and dermal papilla beginning in late (competent) telogen marks a transition point in the growth cycle; noggin binds BMP4 with a 10 - to 15 -fold greater affinity than does BMPR-1A and may actively reduce association of BMP4 with BMPR- $1 \mathrm{~A}$ that is expressed in the secondary hair germ of the telogen follicle [8]. Inhibition of BMP4 binding to BMPR-1A releases local inhibition of the Wnt signaling pathway, leading to up regulation of sonic hedgehog (Shh) and its receptor Patched (Ptc) and is one of the earliest features of hair follicle formation [9].

\section{Activation of the Telogen to Anagen Tranistion}

Coordination of the Wnt/b-catenin and BMP pathways to effect hair follicle regeneration is function not of just what is secreted but also of when and where. Two members of the Tcf/Lef family, Tcf3 and Lef1, are expressed in the hair follicle [10]. Tcf3 expression has been identified in the bulge and in the secondary hair germ, where it appears to function independently of the bcatenin interacting domain to suppress features of epidermal terminal differentiation, thereby maintaining their stem cell features. Lef1, which requires Wnt signaling and b-catenin stabilization to exert regulatory control over hair differentiation, is expressed in the dermal papilla and secondary hair germ in late telogen through early anagen without concomitant expression of Shh [11]. Dermal papilla cells also begin producing Fgf7/10 ligands and BMP inhibitors in late (competent) telogen, 
both of which contribute to the progression of the hair follicle into anagen. Specifically, Fgf7/10 stimulate the secondary hair germ and (to a much lesser extent) the bulge to proliferate through FGFR2-111b binding events. This is in stark contrast to the dermal papilla secretary profile in early (refractory) telogen, at which point FGF18 is predominantly expressed and actively inhibits bulge cells through association with FGFR3.

At the onset of anagen, expression of BMP4 and BMPR-1A are down regulated in the germinative compartment of the hair follicle (i.e., the matrix), leading to a high rate of keratinocyte proliferation [8]. Moreover, in late anagen noggin expression extends from the dermal papilla and cyclic epithelium (i.e., the matrix and precortex) to the surrounding connective tissue. Shh is expressed in unilateral clusters of hair matrix keratinocytes, and Lef1 is observed in the matrix and precortical zone, prompting entry of progenitor cells within these regions into postmitotic hair lineages. Bulge stem cells continue to cycle slowly throughout anagen, but the precise mechanism(s) shielding this cell population from the increasing gradient of stimulatory cues has yet to be determined. However, it is speculated that bulge stem cells sparingly supply cells to extend the outer root sheath and refresh the pool of matrix progenitor cells that terminally differentiate after several proliferative cycles. As a result, the bulge has been deemed the "engine maintaining the (hair growth) process" by Greco et al. in their 2008 Cell Stem Cell journal article (DOI10.1016/j.stem.2008.12.009) [11].

\section{Vitality of the Hair Follicle Bulge Region}

The importance of the bulge in the hair follicle growth cycle cannot be overemphasized. As mentioned previously, cells in the lower portion of the bulge undergo gene expression changes that transform them into the secondary germ cells at the end of catagen. The progeny of multipotent bulge cells generates the new lower anagen follicle in response to the cellular and environmental cues discussed above. However, immune histological studies of alopecic scalps have revealed persistent in filtrates of activated $\mathrm{T}$ cells in the bulge region of the transitional calp, that is, the region of scalp lying at the intersection of balding scalp and hair-retaining scalp [12]. Secondary to this prolonged inflammation, in fundable widen and become blocked by laminated keratin, trichogenic elements are replaced with fibrous tracts, and (critically) anagen follicles are rare. Immune response genes are known to be up regulated in early catagen and throughout telogen in normal hair follicles; the infundibulum is an established early target of acute and transient T- cell mediated-protein expression. Indeed, the formation of desmosomes, the proteins responsible for anchoring telogen "club hairs" in place, is perpetuated via translocation of nuclear factor of activated T cells (NFAT) in to the nucleus of bulge cells during telogen [13]. Clearly activation of the immune response is necessary for maintaining hair cycle homeostasis, yet the mechanistic link between androgens and $\mathrm{T}$ cell dysregulation remains unclear.
Nevertheless, clinical manifestations of androgenic alopecia (AA) support an immune- mediated attack on the bulge. The time course of AA progresses as follows: hair shafts become void of pigmentation; the diameter of individual hair shafts decreases while total hair count remains stable; finally, hair count begins to decline while follicular unit density remains stable. In the hair follicle regeneration cascade, arrest of melanogenesis (i.e., pigment production) precedes that of keratinocyte proliferation, as evidenced by the un pigmented base of the "club" hair in normal telogen. Thus, one may conclude that in the wake of replicative exhaust, depigmentation would be the first clinical manifestation regardless of the source of melanocyte stem cells and matrix transit-amplifying progenitor cells. However, unlike telogen, AA-associated depigmentation extends the entire hair shaft, indicating that transit-amplifying cell populations are continually being replenished by the bulge while melanocyte stem cells, which are located at the base of the bulge, are not. With the passage of time, the bulge region responsible for fueling matrix keratinocyte populations also become compromised, and hair shaft diameter begins to decrease. Once trichogenic elements in the bulge are fully replaced with fibrous tracts, the asynchrony of hair follicle growth becomes apparent. Individual hair follicles within a given follicular unit and receiving progenitor cells from the same bulge region may exist at different states of proliferative potential; therefore, they may reach replicative senescence at significantly later times. That is, if one hair shaft in a follicular unit has been in anagen for 6 years, its matrix cell population may be more "exhausted" than a neighboring hair shaft that has only been in anagen for 6 months. As a result, when the older hair reaches senescence and the fibrous bulge is unable to regenerate the secondary germ during telogen, anagen does not accompany exogen. However, the younger hair may remain intact for several more years since anagen can persist for decades in humans, but follicular unit density will be down, and the growing hair will like lypresent with a small diameter.

\section{Medical Treatments to Combat Hair Loss}

How then can clinicians roll back the clock on a cellular destructive process? The answer may be summed up by the age-old adage; an ounce of prevention is worth a pound of cure. Begin with early intervention. Anti-inflammatory medications such as minoxidil and cyclosporine A would reduce immunemediated "attack" on bulge stem cells by preventing mast cell degranulation. Furthermore, minoxidil is known to exert antiproliferative effects on dermal fibroblasts, decreasing collagen synthesis and there by reducing fibrosis in the susceptible hair loss regions. Experimental stage therapies, including methyl vanillate, aminotic membrane, and WNT-Act, may stimulate anagen-inducing and/or transitional elements. Topical application of methyl vanillate, for example, has been found to increase hair count and hair mass index in women by $6 \%$ and $12 \%$ in women following 6 months of use, respectively. The active 
ingredient is a suspected Wnt-activator given the concomitant $32 \%$ increase in Wnt10B expression in the temporal scalp [14]. Treatment of mice with amniotic membrane has similarly shown up regulation of anagen stimulatory signals, specifically, increased FGF7 and proliferating cell nuclear antigen. Mice treated with topical amniotic membrane expressed similar levels of hair regeneration as those treated with 5\% minoxidil [15]. Additional drug therapies may target extra follicular domains, such as adipocyte precursors whose generation begins in late catagen. Release of platelet derived growth factor (PDGF) from these cells is linked to the suppression of BMP and subsequent on set of anagen. Autologous platelet rich plasma (PRP) is rich in PDGF and has been shown to increase hair density by $50 \%$ at 6 months.

Prescription medications like finasteride have also proven beneficial. Regulation of Wnt signaling in dermal papilla cells has demonstrated an androgen dependence in AA. Dermalpapilla harvested from the scalps of AA patients express increased levels of androgen receptor (AR), which is a member of the nuclear receptor super family that translocates to the nucleus upon binding ligand where it functions as a ligand-dependent transcription factor [16]. Thus, in AA patients, increased AR expression is associated with increased translocation of testosterone-(T) or dihydrotestoster one- (DHT) bound AR. This nuclear complex interacts with b-catenin to inhibit Wntmediated transcriptional activity, and the result is keratinocyte growth suppression in the matrix. Additionally, AR enhanced nuclear translocation of b-cateninin pre-adipocytes, ultimately preventing their differentiation. The sum effect is suppressed hair growth in anagen follicles. Since finasteride is a selective 5a-reductase inhibitor that blocks the conversion of T into DHT (which binds AR with a slightly higher affinity than T [17], the medication may be considered a Wnt up regulator.

Oral finasteride formulations have been associated with increased scalp hair density in men, gaining widespread attention under the trade name Propecia in the late 1990s. However, the medication does not stop the hair loss process and must be taken perpetually to prevent relapse. Since the oral formulation is associated with a number of undesirable side effects (including sexually function), patient compliance is an issue that topical finasteride formulations could conceivably bypass. Although topical formulations are in their infancy of use, remarkable improvements in hair density have been observed, and, in that regard, may represent the best available anti-aging medication for hair at present.

In summary, the factors governing the hair follicle cycle are vast and rather complex. Two inter-related path ways (Wnt/bcatenin and BMP) have been examined in great detail, but the exact trigger mechanism pushing refractory telogen follicles to become competent follicles remains unknown. Extra follicular players, particularly adiposity precursors, appear to be involved. Similarly, androgen sensitivity is a suspected culprit in immune deregulation leading to bulge region fibrosis and eventual demise of the entire hair follicle. Therefore, anti-aging efforts should begin early with anti-inflammatory agents, adipocyte supporters (like PDGF), and Wnt cycle promoters (such as topical finasteride, methyl vanillate, and amniotic membrane).

\section{References}

1. Schneider MR, Schmidt- Ullrich R, Paus R (2009) The Hair Follicleasa Dynamic Miniorgan. Curr Biol 19(3): R132-R142.

2. Lin KK, Kumar V, Geyfman M, (2009) Circadian Clock Genes Contribute to the Regulation of Hair Follicle Cycling. PLoS Genet 5(7): e1000573.

3. Al-Nuaimi Y, Hardman JA, Biro T, et al.A Meeting of Two Chronobiological Systems: Circadian Proteins Period1 and BMAL1 Modulate the Human Hair Cycle Clock. Journal of Investigative Dermatology 3(4): 610-619.

4. Geyfman M, Plikus MV, Treffeisen E, Andersen B, Paus R (2015) Resting no more: re-defining telogen, the maintenance stage of the hair growth cycle. Biol Rev Camb Philos Soc 90(4): 1179-1196.

5. Lowry WE, Blanpain C, NowakJ A, Guasch G, Lewis L, et al. (2005) Defining the impact of beta- catenin/Tcftrans activation on epithelial stem cells. Genes Dev19: 1596-1611.

6. Reya T, Clevers H (2005) Wnt signalling in stem cells and cancer. Nature 434(7035): 843-850.

7. Komiya Y, Habas R (2008) Wht signal transduction pathways. Organogenesis 4(2): 68-75.

8. Botchkarev VA, Botchkareva NV, Nakamura M, Huber O, Funa K, et al. (2001) Noggin is required for induction of the hair follicle growth phase in postnatal skin. FASEB 15: 2205-2214.

9. Lo Celso C, Prowse DM, Watt FM (2004) Transient activation of betacatenin signalling in adult mouse epidermis is sufficient to induce new hair follicles but continuous activation is required to maintain hair follicle tumours. Development 131(8): 1787-1799.

10. Merrill BJ, Gat U, Das Gupta R, Fuchs E (2001) Tcf3 and Lef1 regulate lineage differentiation of multipotent stem cells in skin. Genes \& Development 15(13): 1688-1705.

11. Greco V, Chen T, Rendl M (2010) A two-step mechanism for stem cell activation during hair regeneration. Journal of Investigative Dermatology 130: S102.

12. Jaworsky C, Kligman AM, Murphy GF (1992) Characterization of inflammatory infiltrates in male pattern alopecia - Implications for pathogenesis. Br J Dermatol 127: 239-246.

13. Sato-Miyaoka M, Hisatsune C, Ebisui E, Ogawa N, Takahashi-Iwanaga H, et al. (2012) Regulation of Hair Shedding by the Type 3IP3 Receptor. J Invest Dermatol 132(9): 2137-2147.

14. Tosti A, Zaiac MN, Canazza A (2016) Topical application of the Wnt/ $\beta$ caten inactivator methyl vanillate increases hair count and hair mass index in women with androgentic alopecia. J Cosmet Dermatol 15: 469-474.

15. Seo HS, LeeD-J, ChungJ-H (2016) Hominis Placenta facilitates hair re-growth by up regulating cellular proliferation and expression of fibroblast growth factor-7. BMC Complement Altern Med 16: 187.

16. Kitagawa T, Matsuda K, Inui S (2009) Keratinocyte Growth Inhibition through the Modification of Wnt Signaling by Androgen in Balding Dermal Papilla Cells. J Clin Endocrinol Metab 94(4): 1288-1294.

17. Wilson EM, French FS (1976) Binding properties of androgen receptors - Evidence for identical receptros in rat testis, epididymis, and prostate. J Biol Chem 251(1): 5620-5629. 

(C) Commons Attribution 4.0 License

DOI: 10.19080/IJCSMB.2018.04.555645

\section{Your next submission with Juniper Publishers will reach you the below assets}

- Quality Editorial service

- Swift Peer Review

- Reprints availability

- E-prints Service

- Manuscript Podcast for convenient understanding

- Global attainment for your research

- Manuscript accessibility in different formats

( Pdf, E-pub, Full Text, Audio)

- Unceasing customer service

Track the below URL for one-step submission https://juniperpublishers.com/online-submission.php 